\title{
THE TWO-PIECE PROPERTY AND TIGHT $n$-MANIFOLDS-WITH-BOUNDARY IN $E^{n}$
}

\author{
BY \\ THOMAS F. BANCHOFF( $\left.{ }^{1}\right)$
}

\begin{abstract}
The two-piece property for a set $A$ is a generalization of convexity which reduces to the condition of minimal total absolute curvature if $A$ is a compact 2-manifold. We show that a connected compact 2-manifold-with-boundary in $E^{2}$ has the TPP if and only if each component of the boundary has the TPP. The analogue of this result is not true in higher dimensions without additional conditions, and we introduce a stronger notion called $k$-tightness and show that an $(n+1)$ manifold-with-boundary $M^{n+1}$ embedded in $E^{n+1}$ is 0 - and $(n-1)$-tight if and only if its boundary is 0 - and $(n-1)$-tight.
\end{abstract}

A set $A$ in $E^{n}$ is said to have the two-piece property (TPP) if and only if every hyperplane in $E^{n}$ cuts $A$ into at most two pieces. If $A$ is a smoothly immersed 2-manifold without boundary, then the TPP is equivalent to the condition that $A$ has minimal total absolute curvature in the sense of Chern and Lashof [4]. For an embedded connected 2-manifold-with-boundary $M^{2}$ in $E^{2}$, the TPP for $M^{2}$ is equivalent to the condition that each component of $\partial M^{2}$ has the TPP, but the corresponding result is not correct for higher dimensions. We introduce a finer concept, called $k$-tightness, and we use this to prove an analogue for the 2-dimensional result: If $M^{n+1}$ is an $(n+1)$-manifold-with-boundary embedded in $E^{n+1}$, then $M^{n+1}$ is 0 - and $(n-1)$-tight if and only if $\partial M^{n+1}$ satisfies the same conditions.

An equivalent form of the TPP has been used by the author in several other investigations [1], [2], [3]. A notion similar to $k$-tightness has been introduced by Kuiper in [5], but that treatment uses singular homology while this presentation uses a somewhat stronger idea of what it means for a submanifold $M^{k}$ to bound, i.e., we require that the submanifold $M^{k}$ be the boundary of an embedded $(k+1)$ dimensional manifold-with-boundary. See also Kuiper's article [6] for recent developments in this other direction.

The first section of this paper deals in an elementary way with the case $n=2$. The second section provides the definitions for the higher dimensional investigation and proves a duality theorem for manifolds without boundary, and the final section proves the main theorem mentioned above.

Received by the editors June 17, 1969 and, in revised form, February 4, 1971.

AMS 1970 subject classifications. Primary 53C45, 53C65, 57A17.

Key words and phrases. Tight manifolds, two-piece property, minimal total absolute curvature.

(1) During the period of the writing of this paper, the author was partially supported under the grants NSF GP-9613 and NSF GP-19680.

Copyright (C) 1971, American Mathematical Society 
1. The two-piece property in the plane.

Definition. A set $A$ in $E^{2}$ is said to have the two-piece property (TPP) if every line in $E^{2}$ separates $A$ into at most two pieces.

The entire plane $E^{2}$ has the TPP, since any straight line $l$ determines exactly two open half-planes $\mathrm{H}^{+}(l)$ and $\mathrm{H}^{-}(l)$. Other TPP sets are the empty set, a one-point set, and a set with just two points. This last-mentioned set is the only example which is not arc-connected, where a set $A$ is said to be arc-connected if for every two points $p$ and $q$ of $A$ there is an arc $\gamma$, the 1-1 continuous image of a closed interval, from $p$ to $q$ in $A$. Note that an arc must be embedded.

We shall restrict ourselves in this section to arc-connected sets $A$, and we may make the definition of the TPP more precise as follows:

Definition. An arc-connected set $A$ in $E^{2}$ has the TPP if and only if $\bar{H} \cap A$ is arc-connected for any closed half-plane $\bar{H}$ in $E^{2}$.

Remark 1.1. Any convex set $A$ has the TPP since $A \cap \bar{H}$ will be convex for every $\bar{H}$, and therefore arc-connected.

One example of a nonconvex set with the TPP is a circle. More generally, the following two propositions give a characterization of all curves with the TPP:

Proposition 1.2. If $A$ is a closed curve which is the boundary of a bounded region $R$ in $E^{2}$, then $A$ has the TPP if and only if $R$ is a convex region.

Proof. If $p$ and $q$ are on the boundary $A$ of a convex region $R$, then $p$ and $q$ separate $A$ into two arcs $\gamma_{1}$ and $\gamma_{2}$ such that for any $r$ in $\gamma_{1}$ and $s$ in $\gamma_{2}$, the segment [rs] meets the segment [pq]. If $p$ and $q$ lie in $A \cap \bar{H}$, then either $\gamma_{1}$ or $\gamma_{2}$ lies in $A \cap \bar{H}$, for otherwise we could find $r$ in $\gamma_{1} \cap\left(E^{2}-\bar{H}\right)$ and $s$ in $\gamma_{2} \cap\left(E^{2}-\bar{H}\right)$, and then the segment $[r s]$ would lie in the convex set $E^{2}-\bar{H}$ so the segment [pq], in $\bar{H}$, would not meet the segment $[r s]$, a contradiction.

Conversely, if $\bar{R}$ is a nonconvex bounded connected region with a single boundary curve $A$, then $A$ does not coincide with the boundary $\partial \mathscr{H}(R)$ of the convex hull $\mathscr{H}(R)$ of $R$, i.e., the smallest convex set containing $R$. Therefore there is a segment $[p q]$ of $\partial \mathscr{H}(R)$, lying in a line $l$ bounding a half-plane $\bar{H}^{+}(l)$ containing $\bar{R}$, such that $p$ and $q$ are in $A$ but there is a point $r$ of the segment [pq] not in $A$. But then there is no arc from $p$ to $q$ in $\bar{H}^{-}(l)$, since $A \cap \bar{H}^{-}(l)$ is contained in $l$ and there is no arc from $p$ to $q$ in $A \cap l$, so $A$ does not have the TPP.

Note that the boundary $A$ of a nonbounded region $R$ with the TPP may fail to have the TPP; for example, the convex region $R$ bounded by a parabola has a boundary curve without the TPP.

REMARK 1.3. The proof of the converse in the above proposition establishes the stronger result that a closed set $A$ with the TPP must contain the boundary $\partial \mathscr{H}(A)$ of its convex hull $\mathscr{H}(A)$.

Proposition 1.4. If $A$ is a nonclosed curve in $E^{2}$, with or without endpoints, then $A$ has the TPP if and only if $A$ is a connected subset of a line. 
Proof. Any connected subset of a line is convex, so it has the TPP. Conversely, if $p$ and $q$ are in $A$ and the segment [ $p q]$ does not lie in $A$, then at least one of the half-planes $H^{+}(l)$ determined by the line $l$ through $p$ and $q$ contains a point $r$ of the arc of $A$ bounded by $p$ and $q$ so there is no arc from $p$ to $q$ in $\bar{H}^{-}(l) \cap A$ and $A$ does not have the TPP.

Another example of a nonconvex set with the TPP is an annulus, or a disc with several disjoint discs removed, as demonstrated by the following:

Proposition 1.5. If $A$ is a closed convex set and if $\left\{B_{i}\right\}, i=1,2, \ldots, m$, is a finite collection of bounded open convex sets with disjoint boundaries, then $A-\bigcup_{i=1}^{m} B_{i}$ has the TPP.

Proof. Let $p$ and $q$ be points of $A \cap \bar{H}$, so that the segment [pq] will lie in the convex set $A \cap \bar{H}$. If the segment [pq] meets the component $B_{i}$ in the segment $\left[p_{i} q_{i}\right.$ ], then by Proposition 1.2 above, at least one of the arcs $\gamma_{i}$ from $p_{i}$ to $q_{i}$ on $\partial B_{i}$ lies in $\bar{H}$ so we may obtain an embedded path $\gamma$ from $p$ to $q$ in $\left(A-\bigcup_{i=1}^{m} B_{i}\right) \cap \bar{H}$ by replacing each of the segments $\left[p_{i} q_{i}\right]$ by the disjoint arcs $\gamma_{i}$.

REMARK 1.6. Although we do not need a stronger form of this proposition in our investigation of 2-manifolds-with-boundary, the above proposition is true even without the hypothesis that the boundaries of the convex sets be disjoint, for example if $A$ is the complement of a union of (externally) tangent discs, or if $A$ is the union of a circle and a chord. The same proof provides a path $\gamma$ (i.e., the continuous image of a segment) joining $p$ to $q$ in $\left(A-\bigcup_{i=1}^{m} B_{i}\right) \cap \bar{H}$, but if the boundaries $\partial B_{i}$ intersect, then $\gamma$ may turn out not to be an embedding. In this case, however, we may modify the path $\gamma$ to produce an arc $\gamma^{\prime}$ from $p$ to $q$ by proceeding inductively to remove the (finitely many) intersections of the arcs $\gamma_{i}$ as follows: if $\gamma_{i}$ meets $\gamma_{j}$ in the segment $(a, b)$, replace the subpath of $\gamma$ from $p_{i}$ to $q_{j}$ by the union of the subarcs $\left(p_{i} a\right)$ on $\gamma_{i}$ and $\left(a q_{j}\right)$ on $\gamma_{j}$. The same result may be obtained by appealing to the rather more subtle result that any path joining $p$ to $q$ contains an $\operatorname{arc}$ from $p$ to $q$.

REMARK 1.7. Without the assumption that the collection $\left\{B_{i}\right\}$ is finite, the set $A-\bigcup B_{i}$ may fail to have the TPP even if the boundaries of the convex bounded open sets $B_{i}$ are disjoint. For example, the set $A-\bigcup B_{i}$ might fail to be pathconnected, e.g., if $A=E^{2}$ and $B_{i}$ is the open rectangle with vertices $(1 / 2 i, i)$, $(1 /(2 i+1), i),(1 / 2 i,-i),(1 /(2 i+1),-i)$, or the set $\gamma$ constructed in the proof of Proposition 1.4 might fail to be a (continuous) path, e.g., if $A$ is the square with vertices $(-2,-2),(-2,2),(2,2),(2,-2)$ and $B_{i}$ is the rectangle with vertices $(1 / 2 i, 1),(1 /(2 i+1), 1),(1 / 2 i,-1),(1 /(2 i+1),-1)$ (compare [3] where the situation with the "circle TPP" turns out quite differently).

We now establish a strong converse to Proposition 1.5.

THEOREM 1.8. If $A$ is a closed set in $E^{2}$ with the TPP, then each of the bounded components $B_{i}$ of $E^{2}-A$ is a convex set and the union $A \cup \cup B_{i}$ of $A$ and the bounded components of its complement is a convex set. 
Proof. If a bounded component $B_{i}$ of the complement of $A$ is not convex we may find a pair of points of $B_{i}$ such that the segment [pq] contains a point $a$ of $A$. Since $B_{i}$ is bounded, there is a point $b$ of $a$ in $l-(\operatorname{segment}[p q])$ where $l$ is the line determined by the segment [pq]. Since $A$ has the TPP there must be an arc $\gamma^{+}$joining $a$ to $b$ in $\bar{H}^{+}(l) \cap A$ and another arc $\gamma^{-}$joining $a$ to $b$ in $\bar{H}^{-}(l) \cap A$. If $\gamma^{+} \cap \gamma^{-}$ $=\{a, b\}$, then $\gamma^{+} \cup \gamma^{-}$gives a closed arc in $E^{2}$ which crosses the segment [pq] in just one point (either $a$ or $b$ ) so by the Jordan curve theorem, any arc from $p$ to $q$ must contain a point of $\gamma^{\prime} \cap \gamma^{\prime \prime}$ which is contained in $A$. Therefore $p$ cannot be connected to $q$ in $B_{i}$, contradicting the fact that $B_{i}$ is a component of $E^{2}-A$.

If $\gamma^{+} \cap \gamma^{-}$contains points of $l$ other than $a$ and $b$, it is necessary to make the construction somewhat more subtle. The arc $\gamma^{+}$must contain at least one subarc $\gamma^{\prime}$ from a point $a^{\prime}$ of $A \cap \operatorname{segment}$ [pq] to a point $b^{\prime}$ of $A \cap(l-$ segment [pq]) such that $\gamma^{\prime} \cap l=\left\{a^{\prime}, b^{\prime}\right\}$, and since $A$ has the TPP, there must be an arc $\gamma^{\prime \prime}$ from $a^{\prime}$ to $b^{\prime}$ in $\bar{H}^{-}(l)$, so $\gamma^{\prime} \cap \gamma^{\prime \prime}=\{a, b\}$. Then $\gamma^{\prime} \cup \gamma^{\prime \prime}$ provides a closed arc which still separates $p$ from $q$ since there are arcs $\zeta^{\prime}$ and $\zeta^{\prime \prime}$ from $p$ to $a$ and from $a$ to $q$ near the segment $[p q]$ lying, except for their endpoints, in $H^{+}(l)-\gamma^{\prime}$ and then $\zeta^{\prime} \cup \zeta^{\prime \prime}$ gives an arc from $p$ to $q$ crossing $\gamma^{\prime} \cup \gamma^{\prime \prime}$ at just one point.

We now show that $A \cup \cup B_{i}=\mathscr{H}(A)$, the convex hull of $A$. Otherwise there is a segment [pq] with endpoints in $A$ containing a point $r$ of an unbounded component of the complement $A$, i.e., such that $r$ is contained in no region bounded by a closed arc in $A$. But since $A$ has the TPP we may find $\gamma^{+}$in $\bar{H}^{+}(l) \cap A$ and $\gamma^{-}$ in $\bar{H}^{-}(l) \cap A$ connecting $p$ to $q$, and either $\gamma^{+} \cup \gamma^{-}$already provides a closed arc bounding a disc containing $r$ or we may modify $\gamma^{+}$as in the previous paragraph to find $\gamma^{\prime}$ in $\bar{H}^{+}(l) \cap A$ joining points $a$ and $b$ of different components of $l-\{r\}$ and such that $\gamma^{\prime} \cap l=\{a, b\}$. We may then take $\gamma^{\prime} \cup \gamma^{\prime \prime}$ for an arc $\gamma^{\prime \prime}$ joining $a$ to $b$ in $\bar{H}^{-}(l) \cap A$ to get a closed arc in $A$ bounding a region containing $r$, contradicting the assumption that $r$ was a point of an unbounded component of the complement of $A$.

Up to this point we have carried out our discussion in the spirit of the point-set topology of $E^{2}$, placing very few restrictions on the sets under consideration. In the higher-dimensional situation, however, we will want to make some simplifying assumptions and we now recast our results in this context.

Definition. A 2-manifold-with-boundary $M^{2}$ embedded in $E^{2}$ is a closed point set $M^{2}$ which can be expressed as a union of two sets: $\dot{M}^{2}$, the interior of $M^{2}$ $=\left\{p\right.$ in $M^{2}$ such that there is a disc neighborhood $B^{2}$ of $p$ in $E^{2}$ with $\left.B^{2} \subset M^{2}\right\}$ and $\partial M^{2}=$ the boundary of $M^{2}=\left\{q\right.$ in $M^{2}$ such that there is a neighborhood $B^{2}$ of $q$ in $E^{2}$ with $B^{2} \cap M^{2}$ given by the 1-1 continuous image of the intersection of an open disc about the origin with the closed upper half-plane, with $q$ corresponding to the origin\}.

The boundary $\partial M^{2}$ of a 2-manifold-with-boundary embedded in $E^{2}$ is either empty or the union of a collection of disjoint simple closed curves and simple infinite curves. Moreover, for any closed interval the segment $[p q]$ in $E^{2}$, there can 
be at most a finite number of boundary components of $M^{2}$ meeting the segment [pq], for otherwise there would be an accumulation point $r$ in $A$ of the points $\left\{a_{i}\right\}$, with $a_{i} \in B_{i} \cap$ the segment $[p q]$, contradicting the fact that $r$ has a neighborhood meeting at most one boundary component of $M^{2}$. Therefore the construction in Proposition 1.5 goes through if $A-\cup B_{i}$ is a 2-manifold-with-boundary, even if there are infinitely many components $B_{i}$.

We may then state a theorem summing up all of our results for the case of 2manifolds-with-boundary in $E^{2}$.

THEOREM 1.9. A 2-manifold-with-boundary $M^{2}$ embedded in $E^{2}$ has the TPP if and only if each bounded component of $\partial M^{2}$ has the TPP and if $M^{2} \cup\left(\cup B_{i}\right)$ is a convex set.

2. The higher dimensional case-examples and definitions. In the first section of this paper, the main result implies that a 2-manifold-with-boundary $M^{2}$ in $E^{2}$ has the TPP if and only if each of the components of its boundary $\partial M^{2}$ has the TPP. For 3-manifolds-with-boundary in $E^{3}$, however, the corresponding result is false, as is shown by the following example:
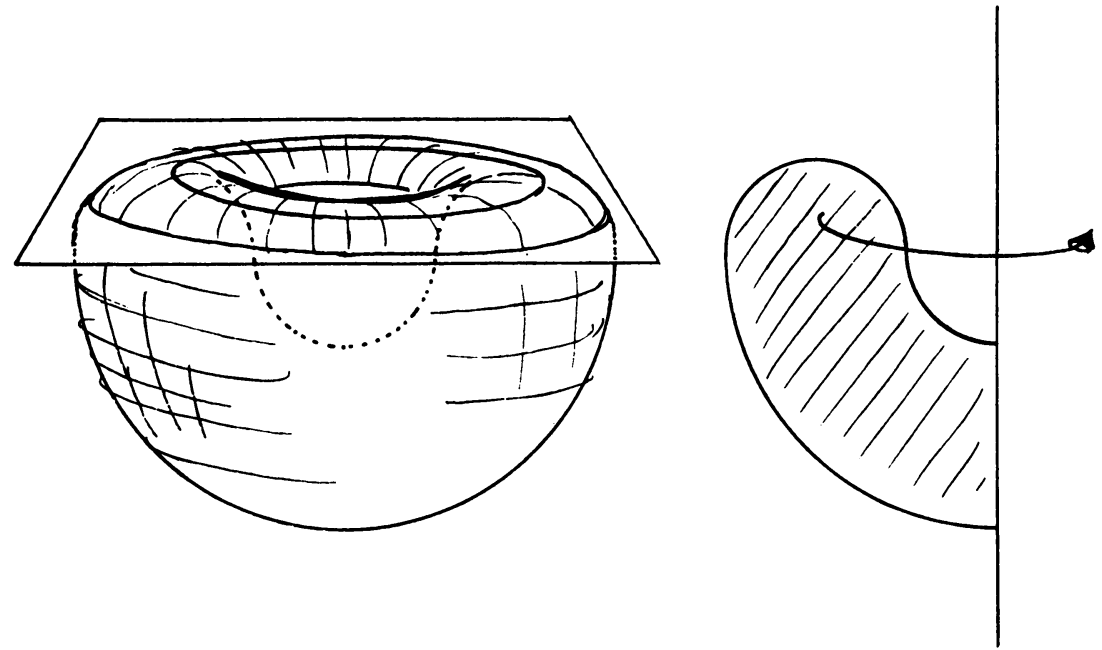

The intersection of the solid figure with any half-space is connected, but a horizontal plane $\pi$ can divide the boundary surface into more than two pieces.

The "problem" in a sense is that although every pair of points in the figure above the plane $\pi$ can be joined by an arc, there is a circle (the top circle) in the figure which bounds a topological disc in the figure but which does not bound any disc in the figure and lying above the plane $\pi$. In the terminology which we shall introduce in this section, the figure is said to be 0 -tight but not 1-tight. We shall prove ultimately that if $M^{3}$ is a 3-manifold-with-boundary embedded in $E^{3}$, then each component of $\partial M^{3}$ has the TPP if and only if each component of $M^{3}$ has the TPP and if $M^{3}$ is 1-tight. 
The results in this section are stated in terms of $(n+1)$-manifolds-with-boundary in $E^{n+1}$, but the reader may safely think of the case $n=2$ in order to keep the geometric meaning clear.

Definition. A $k$-manifold-with-boundary $N^{k}$ embedded in a Euclidean space $E^{n}$ is a closed subset which can be expressed as a union of $\stackrel{\circ}{N}^{k}=$ interior of $N^{k}=\{p$ in $N$ with a neighborhood $B^{n}$ in $E^{n}$ with $B^{n} \cap N^{k}$ homeomorphic to a $k$-dimensional disc $\}$ and $\partial N^{k}=$ boundary of $N^{k}=\left\{q\right.$ in $N$ with a neighborhood $B^{n}$ such that $B^{n} \cap N^{k}$ is homeomorphic to the intersection of the open unit $k$-disc with the closed upper half-space in $E^{k}$, with $q$ corresponding to the origin\}.

We shall work for the most part with compact $k$-manifolds-with-boundary $N^{k}$ and we shall make the strong assumption that almost every hyperplane $\pi$ in $E^{n}$ cuts $N^{k}$, if at all, in a $(k-1)$-manifold-with-boundary embedded in $E^{n}$, i.e., arbitrarily close to any hyperplane $\pi$, there is a parallel hyperplane $\pi^{\prime}$ with the desired property. (This situation occurs for example whenever $N^{k}$ is a smooth or polyhedral submanifold of $E^{n}$.)

Furthermore, we may assume that if $N^{k}$ is a $k$-manifold-with-boundary embedded in $E^{n}$ contained in an $l$-manifold-with-boundary $M^{l}$ with $\partial N^{k}$ contained in $\partial M^{l}$, then there is a $k$-manifold-with-boundary $N^{k}$ such that $\partial \underline{N}^{k}=\partial N^{k}$ and $\stackrel{\circ}{N}^{k}$ is contained in $\stackrel{M}{ }^{l}$, so for example we may "push arcs away from the boundary". (This property is again true for smooth or polyhedral submanifolds, and in all cases when the containing manifold $M^{l}$ is "collared".)

Definition. A set $A$ in $E^{n}$ is called $k$-tight if whenever $N^{k}$ is a $k$-manifold embedded in $E^{n}$ contained in $\bar{H}^{+}(\pi) \cap A$ and also $N^{k}=\partial P^{k+1}$ for some $(k+1)$ manifold-with-boundary $P^{k+1}$ in $A$, then $N^{k}=\partial Q^{k+1}$ for some $(k+1)$-manifoldwith-boundary in $\bar{H}^{+}(\pi) \cap A$.

REMARK. In the terminology of the previous section, $A$ is 0 -tight if and only if each component of $A$ has the TPP.

By the Jordan-Brouwer Separation Theorem, if $N^{k}$ is a connected $k$-manifold without boundary embedded in $E^{k+1}$, then $E^{k+1}-N^{k}$ consists of two components, both $(k+1)$-manifolds-with-boundary with $N^{k}$ as their common boundary. An arc $\gamma$ is said to cross $N^{k}$ at $q$ if there is an open subarc ( $\left.p r\right)$ of $\gamma$ with ( $\left.p q\right)$ in one component of $E^{k+1}-N^{k}$ and ( $\left.q r\right)$ in the other. If $\gamma$ is a closed arc which crosses $N^{k}$ a finite number of times, then the separation theorem implies that $\gamma$ crosses $N^{k}$ an even number of times.

If $N^{k}$ is a $k$-manifold without boundary, possibly not connected, embedded in $E^{k+1}$, then again we may express $E^{k+1}-N^{k}$ as a union of two $(k+1)$-manifoldswith-boundary, $C_{1}^{k+1}\left(N^{k}\right)$ and $C_{2}^{k+1}\left(N^{k}\right)$, possibly not connected, with $N^{k}$ as their common boundary, in such a way that an arc $\gamma$ joining points $p$ and $q$ in $E^{k+1}-N^{k}$ crosses $N^{k}$ an even number of times if and only if $p$ and $q$ lie in the same one of these $(k+1)$-manifolds-with-boundary.

Furthermore, if $N^{k}$ is a $k$-manifold without boundary embedded in any $(k+1)-$ manifold $M^{k+1}$ without boundary, and if $N^{k}$ bounds a $(k+1)$-manifold-with- 
boundary $P^{k+1}$ embedded in $M^{k+1}$, then it follows that $Q^{k+1}=M^{k+1}-P^{k+1}$ is also a $(k+1)$-manifold-with-boundary embedded in $M^{k+1}$ with the same boundary $N^{k}$.

REMARK 2.1. In the definition of $k$-tightness we make use of closed half-spaces but in certain arguments we wish to make use of open half-spaces as well. For example, if $M^{n} \cap \bar{H}^{+}(\pi)$ consists of more than one component, then so do $M^{n} \cap \bar{H}^{+}\left(\pi^{\prime}\right)$ and $M^{n} \cap H^{+}\left(\pi^{\prime}\right)$ for some hyperplane $\pi^{\prime}$ parallel to $\pi$ and close to $\pi$. We use this remark in the proof of the following duality theorem:

THEOREM 2.2. If $M^{k}$ is a $k$-manifold (without boundary) embedded in $E^{n}$, then $M^{k}$ is 0-tight if and only if $M^{k}$ is $(k-1)$-tight.

Proof. It suffices to prove that each component of $M^{k}$ is $(k-1)$-tight if and only if it has the TPP, so we shall assume that $M^{k}$ is connected.

If $M^{k}$ is $(k-1)$-tight but not 0 -tight, then there is a hyperplane $\pi$ such that $M^{k} \cap \bar{H}^{+}(\pi)$ consists of more than one component, and, by the previous remark, we may assume that $M^{k} \cap H^{+}\left(\pi^{\prime}\right)$ consists of a component $L^{k}$ and at least one other component, and also that $\partial L^{k}=N^{k-1}$ is a $(k-1)$-manifold. Since $N^{k-1}$ lies in $M^{k} \cap \bar{H}^{-}\left(\pi^{\prime}\right)$ and bounds in $M^{k}$, and since $M^{k}$ is $(k-1)$-tight, there must be a $k$-manifold-with-boundary $P^{k}$ in $M^{k} \cap \bar{H}^{-}\left(\pi^{\prime}\right)$ with $\partial P^{k}=N^{k-1}$. But then $P^{k} \cup L^{k}$ gives a $k$-manifold embedded in $M^{k}$, so $P^{k} \cup L^{k}=M^{k}$, contradicting the assumption that there were points of $H^{+}\left(\pi^{\prime}\right)$ not in $L^{k}$.

Conversely, if $M^{k}$ is 0 -tight and $N^{k-1}$ is a $k$-manifold without boundary in $M^{k} \cap \bar{H}^{+}(\pi)$ which bounds a $k$-manifold-with-boundary $P^{k}$ in $M^{k}$, then $N^{k-1}$ $=\partial\left(M^{k}-\ddot{P}^{k}\right)$ as well, and it suffices to prove that either $P^{k}$ or $Q^{k}=M^{k}-\dot{P}^{k}$ lies in $\bar{H}^{+}(\pi)$. Otherwise, there would be points $p$ and $q$ in $H^{-}\left(\pi^{\prime}\right) \cap P^{k}$ and $H^{-}\left(\pi^{\prime}\right) \cap Q^{k}$ respectively, where $\pi^{\prime}$ is parallel to $\pi$ and where $N^{k-1}$ lies in $H^{+}\left(\pi^{\prime}\right)$. Since any arc from a point in $P^{k}$ to a point in $Q^{k}$ must cross $N^{k-1}$ in an odd number of points, there is no arc from $p$ to $q$ in $\bar{H}^{-}\left(\pi^{\prime}\right)$, contradicting the hypothesis that $M^{k}$ is 0-tight.

3. The main theorem for $(n+1)$-manifolds-with-boundary in $E^{n+1}$. In the remainder of this paper, we wish to study the relationship between the $k$-tightness of an $n$-manifold-with-boundary embedded in $E^{n}$ and the $k$-tightness of its boundary. The first result is analogous to the 2-dimensional case:

THEOREM 3.1. If $M^{k+1}$ is an $(n+1)$-manifold-with-boundary embedded in $E^{n+1}$ and if $\partial M^{n+1}$ is 0 -tight, then so is $M^{n+1}$.

Proof. If $p$ and $q$ are points of $M^{n+1} \cap \bar{H}^{+}(\pi)$ that can be joined by an arc $\gamma$ in $\dot{M}^{n+1}$ except perhaps for $p$ and $q$, then consider any arc $\gamma^{\prime}$ from $p$ to $q$ in $\bar{H}^{+}(\pi)$ which crosses $\partial M^{n+1}$ a finite number of times (any arc meets only finitely many components of $\left.\partial M^{n+1}\right)$. Such an arc $\gamma^{\prime}$ crosses any component $B_{i}^{n}$ of $\partial M^{n+1}$ an even number of times since $\gamma \cup \gamma^{\prime}$ is a closed arc and $\gamma \cap B_{i}^{n}=\varnothing$. Then if $p_{i}$ and $q_{i}$ denote the first and last crossing points on the path $\gamma^{\prime}$ from $p$ to $q$, there is a new 
path $\gamma_{i}$ from $p_{i}$ to $q_{i}$ in $B_{i}^{n} \cap \bar{H}^{+}(\pi)$ since $\partial M^{n+1}$ is assumed to be 0 -tight, and we may replace the subarc from $p_{i}$ to $q_{i}$ on $\gamma^{\prime}$ by $\gamma_{i}$ for each component $B_{i}^{n}$ of $\partial M^{n+1}$ to obtain a new arc $\gamma^{\prime \prime}$ from $p$ to $q$ in $M^{n+1} \cap \bar{H}^{+}(\pi)$ as required.

THEOREM 3.2. If $M^{n+1}$ is an $(n+1)$-manifold-with-boundary embedded in $E^{n+1}$ and if $\partial M^{n}$ is 0-tight, then $M^{n+1}$ is $(n-1)$-tight.

Proof. If $N^{n-1}$ is an $(n-1)$-manifold in $M^{n+1} \cap \bar{H}^{+}(\pi)$ which bounds an $n$-manifold-with-boundary $P^{n}$ in $M^{n+1}$, we may assume that $P^{n} \cap M^{n+1}=\varnothing$ and that $P^{n}$ crosses $\pi$ in an $(n-1)$-manifold $Q^{n-1}=P^{n} \cap \pi$. Then consider the $n$ manifolds-with-boundary $C_{1}^{n}\left(Q^{n-1}\right)$ and $C_{2}^{n}\left(Q^{n-1}\right)$ in $\pi$. If $\partial M^{3} \cap C_{i}^{n}\left(Q^{n-1}\right)=\varnothing$ for one of these $n$-manifolds-with-boundary, then $\left(P^{n} \cap \bar{H}^{+}(\pi)\right) \cup C_{i}^{n}\left(Q^{n-1}\right)$ provides an $n$-manifold-with-boundary in $M^{n} \cap \bar{H}^{+}(\pi)$ with boundary $N^{n-1}$ as required.

Furthermore, if $R^{n}$ is the union of all of the components of $\partial M^{n+1}$ which meet $C_{1}^{n}\left(Q^{n-1}\right)$ and if none of these components meets $C_{2}^{n}\left(Q^{n-1}\right)$, we may set

$$
U^{n}=\left(P^{n} \cap \bar{H}^{+}(\pi)\right) \cup C_{1}^{n}\left(Q^{n-1} \cup\left(R^{n} \cap \pi\right)\right) \cup\left(R^{n} \cap \bar{H}^{+}(\pi)\right)
$$

to get an $n$-manifold-with-boundary in $\bar{H}^{+}(\pi) \cap M^{n+1}$ with boundary $N^{n-1}$ as required.

To complete the proof we need only show that if a component $B_{i}^{n}$ of $\partial M^{n+1}$ meets $C_{1}^{n}\left(Q^{n-1}\right)$, then it does not meet $C_{2}^{n}\left(Q^{n-1}\right)$. For otherwise we may find an arc $\gamma$ in $B_{i}^{n} \cap \bar{H}^{+}(\pi)$ from a point $p$ in $B_{i}^{n} \cap C_{1}^{n}\left(Q^{n-1}\right)$ to $q$ in $B_{i}^{n} \cap C_{2}^{n}\left(Q^{n-1}\right)$, and an arc $\gamma^{\prime}$ from $p$ to $q$ in $B_{i}^{n} \cap \bar{H}^{-}(\pi)$ since we have assumed that $\partial M^{n+1}$ is 0 -tight. But then $\gamma \cup \gamma^{\prime}$ is a closed arc which crosses at just the point $p$ the $n$-manifold without boundary $\left(P^{n} \cap \bar{H}^{-}(\pi)\right) \cup C_{1}^{n}\left(Q^{n-1}\right)$, a contradiction.

Before proceeding to the final step in the main theorem of this section, we establish a lemma.

LEMMA 3.3. If $B^{n}$ is a component of the boundary $\partial M^{n+1}$ of a 0-tight $(n+1)$ manifold-with-boundary $M^{n+1}$ embedded in $E^{n+1}$, and if $B^{n} \cap \bar{H}^{+}(\pi)$ is an $n$ manifold-with-boundary containing at least two components $P^{n}$ and $Q^{n}$, then $Q^{n}$ cannot meet both $C_{1}^{n}\left(P^{n} \cap \pi\right)$ and $C_{2}^{n}\left(P^{n} \cap \pi\right)$.

Proof. Otherwise we could find an arc $\gamma$ from a point $p$ of $Q^{n} \cap C_{1}^{n}\left(P^{n} \cap \pi\right)$ to a point $q$ of $Q^{n} \cap C_{2}^{n}\left(P^{n} \cap \pi\right)$ with $\gamma$ lying in $Q^{n} \cap \bar{H}^{+}(\pi)$ and an arc $\gamma^{\prime}$ from $p$ to $q$ in $\dot{M}^{n+1} \cap \bar{H}^{-}(\pi)$ except for its endpoints since $M^{n+1}$ is 0 -tight. But then $\gamma \cup \gamma^{\prime}$ is a closed arc which crosses the $n$-manifold without boundary $P^{n} \cup C_{1}^{n}\left(P^{n} \cap \pi\right)$ at exactly one point $p$, a contradiction.

THEOREM 3.4. If $M^{n+1}$ is an $(n+1)$-manifold-with-boundary embedded in $E^{n+1}$ which is 0 - and (n-1)-tight, then $\partial M^{n+1}$ is 0-tight.

Proof. If $\partial M^{n+1}$ is not 0 -tight, then there is a component $B^{n}$ of $\partial M^{n+1}$ and a hyperplane $\pi$ such that $B^{n} \cap \bar{H}^{+}(\pi)$ is an $n$-manifold-with-boundary consisting of 
at least two components. Let $P^{n}$ and $Q^{n}$ be two such components such that there is an arc $\gamma$ in $B^{n} \cap \bar{H}^{-}(\pi)$ joining a point $p$ in $P^{n} \cap \pi$ to $q$ in $Q^{n} \cap \pi$. Since $M^{n+1}$ is 0-tight, there is an arc $\gamma^{\prime}$ from $p$ to $q$ in $\dot{M}^{n+1} \cap \bar{H}^{+}(\pi)$ except for its endpoints $p$ and $q$. By the previous lemma, $Q^{n}$ will not meet both $C_{1}^{n}\left(P^{n} \cap \pi\right)$ and $C_{2}^{n}\left(P^{n} \cap \pi\right)$, so we may assume that $Q^{n} \cap C_{2}^{n}\left(P^{n} \cap \pi\right)=\varnothing$. We then have two cases to consider. If $C_{2}^{n}\left(P^{n} \cap \pi\right)$ contains points of $\dot{M}^{n+1}$, then $\gamma \cup \gamma^{\prime}$ crosses the $n$-manifold without boundary $\left(P^{n} \cap \bar{H}^{+}(\pi)\right) \cup\left(C_{2}^{n}\left(P^{n} \cap \pi\right)\right)$ exactly once at $p$, a contradiction. If $C_{2}^{n}\left(P^{n} \cap \pi\right)$ does not contain points of $M^{n+1}$, then since $M^{n+1}$ is assumed to be (n-1)-tight there is an $n$-manifold-with-boundary $R^{n}$ in $\dot{M}^{n+1} \cap \bar{H}^{-}(\pi)$ except for its boundary and $\partial R^{n}=\left(P^{n} \cap \pi\right)$. Then the closed arc $\gamma \cup \gamma^{\prime}$ crosses the $n$ manifold $\left(P^{n} \cap \bar{H}^{+}(\pi)\right) \cup R^{n}$ at the one point $p$, again a contradiction to the Jordan-Brouwer separation theorem.

REMARK. In the preceding discussion it was not necessary at any stage to assume that the manifolds $M^{n+1}$ or any of its boundary components were compact. If compactness is assumed, then some of the proofs can be simplified.

\section{BIBLIOGRAPHY}

1. T. F. Banchoff, High codimensional 0-tight maps on spheres, Proc. Amer. Math. Soc. 29 (1971), 133-135.

2. - - Tightly embedded 2-dimensional polyhedral manifolds, Amer. J. Math. 87 (1965), 462-472. MR 31 \#2729.

3. - - The spherical two-piece property and tight surfaces in spheres, J. Differential Geometry 4 (1970), 193-205.

4. S. Chern and R. Lashof, On the total curvature of immersed manifolds. I, Amer. J. Math. 79 (1957), 306-318. MR 18, 927.

5. N. H. Kuiper, On convex maps, Nieuw Arch. Wisk. (3) 10 (1962), 147-164. MR 26 \#3076.

6. - Minimal total absolute curvature for immersions, Invent. Math. 10 (1970), 209-238.

Brown University, Providence, Rhode Island 02912 